\title{
A sharp Trudinger type inequality for harmonic functions and its applications
}

Yili Tan ${ }^{1}$, Yongli An², Hong Wang ${ }^{1}$ and Jing Liü ${ }^{3 *}$

${ }^{\text {"Correspondence: }}$

3448043660@qq.com

${ }^{3}$ Department of Basic Courses, Zhejiang New Century College of

Economy and Trade, Hangzhou,

311122, China

Full list of author information is

available at the end of the article

\begin{abstract}
The present paper introduces a sharp Trudinger type inequality $r$ harmonid functions based on the Cauchy-Riesz kernel function, which incl s modified Poisson type kernel in a half plane considered by Xu et al ' $\mathrm{Bc}$ d. Varue Probl. 2013:262, 2013). As applications, we not only obtain M rrey rep. sntations of continuous linear maps for harmonic functions in t' es of all closed bounded convex nonempty subsets of any Banach space hit also a "acke the representation for set-valued maps and for scalar-valued ma is ot unford-schwartz.
\end{abstract}

Keywords: Trudinger type inequality; Cauchy ssz kernel function; modified Poisson type kernel; Morrey representa

\section{Introduction}

The Trudinger inequal; cy prob $\eta$ (IIP) is generated from the method of mathematical physics and nonl. ar rograzmming. It has considerable applications in many fields such as physics, mechan fngineering, economic decision, control theory and so on. Trudinger in qua $_{\text {a }}$ is actually a system of partial differential equations. Especially, physicists have iong been ing so-called singular functions such as the Dirac delta function $\delta$, althoug these cannot be properly defined within the framework of classical function theory. The irac d lta function $\delta(x-\xi)$ is equal to zero everywhere except at a fixed point $\xi$. A rding to the classical definition of a function and an integral, these conditions are inconsiste,, . In elementary particle physics, one found the need to evaluate $\delta^{3}$ when calculatir $\sigma$ the transition rates of certain particle interactions [2]. In [3], a definition of product distributions was given using delta sequences. In [4], Bremermann used the Cauchy representations of distributions with compact support to define $\sqrt{\delta_{+}}$and $\log \delta_{+}$. Unfortunately, his definition did not carry over to $\sqrt{\delta}$ and $\log \delta$. In 1964, Gel'fand and Shilov [5] defined $\delta^{(k+1)}(P)$ for an infinitely differentiable function $P\left(x_{1}, x_{2}, \ldots, x_{n}\right)$ such that the $P=0$ hypersurface had no singular points, where

$$
P=P\left(x_{1}, x_{2}, \ldots, x_{p+q}\right)=x_{1}^{2}+x_{2}^{2}+\cdots+x_{p}^{2}-x_{p+1}^{2}-\cdots-x_{p+q}^{2},
$$

$p+q=n$ is the dimension of the Euclidean space $\mathbb{R}^{n}$, the $P=0$ hypersurface was a hypercone with a singular point (the vertex) at the origin. Then they also defined the generalized functions $\delta_{1}^{(k+1)}(P)$ and $\delta_{2}^{(k+1)}(P)$ as in the cases $p, q<1$ and $p, q=1$, respectively. By the Sobolev embedding theorem, it was well known that the Sobolev space $H^{1}(G)$

(c) The Author(s) 2017. This article is distributed under the terms of the Creative Commons Attribution 4.0 International License (http://creativecommons.org/licenses/by/4.0/), which permits unrestricted use, distribution, and reproduction in any medium, provided you give appropriate credit to the original author(s) and the source, provide a link to the Creative Commons license, and indicate if changes were made. 
was embedded in all Lebesgue spaces $L^{p}(G)$ for $2<p<\infty$ but not in $L^{\infty}(G)$. Moreover, $\delta_{1}^{(k)}(P)$ and $\delta_{2}^{(k)}(P)$ functions were in the so-called Orlicz space, i.e., their exponential powers were integrable functions. Precisely, Ruf established the Trudinger inequality (see [6, Theorem 2.1]). However, the best possible constant $\beta$ in it was much more interesting and was not exhibited until the 2008 paper [7] of Li and Ruf. In fact, using the symmetrization argument to reduce to the one-dimensional case, they established a result which is now called the Trudinger inequality. It was refined and extended to many different settings. For instance, a singular Trudinger inequality which was an interpolation of Hard inequality and Trudinger inequality was studied by Su in [8]. Meanwhile, Su further studied the residue of the generalized function $G^{\lambda}$, where $\lambda$ was a nonnegative real umber. Very recently, Yan et al. [9] have succeeded to establish the sharp constants and tremal functions of the Trudinger inequality on the Heisenberg group and genera. tributional product of Dirac's delta in a hypercone. Furthermore, Li a d Vetro a much simpler method of deriving the product $f(r-1) \cdot \delta^{(k+1)}(r+1)$ fo. II nonnegative integer $k$ and $r=\left(x_{1}^{2}+x_{2}^{2}+\cdots+x_{p+q}^{2}\right)^{1 / 3}$. And they found the pr $\left.\sim P^{n} \cdot \delta^{\prime}\right)^{1 /}(P)$ as well as a general product $f(P) \cdot \delta^{(k+1)}(P)$, where $f$ was a $C_{1}^{\infty}$-functic nn the other study of the products of particular distributions and the development of $\mathrm{c}$ rs' works can be seen in $[1,11]$.

By using augmented Riesz decomposition methods deve oped by Xie and Viouonu [12], the purpose of this paper is to obtain a sharp $\mathrm{T}$ ger typ $\mathrm{e}$ inequality for harmonic functions based on a Cauchy-Riesz kernel func and udy the product $G^{l}(P) \cdot \delta^{(k+1)}(P)$ and then study a more general product of $f\left(F^{\prime}\right) \cdot \delta^{\prime} \quad(P)$, where $f$ is a $C_{1}^{\infty}$-function on $\mathbb{R}$ and $\delta^{(k+1)}(G)$ is the Dirac delta function $n \quad k$ derlvacives. As applications, we not only obtain Morrey representations of cont huous $\mathrm{L} . \mathrm{r} /$ maps for harmonic functions in the set of all closed bounded convex non $\mathrm{m}_{\mathrm{P}}$ subsets of any Banach space, but also deduce the representation for set-valuf u maps ana or scalar-valued maps of Dunford-Schwartz. Before proceeding to our mai results, the following definitions and concepts are required.

\section{Preliminarie}

Definition $9.1 \mathrm{Le}+x=\left(x_{1}, x_{2}, \ldots, x_{n}\right)$ be a point in $\mathbb{R}^{n}$, where $\mathbb{R}^{n}$ is the $n$-dimensional Euclidear pac The ypersurface $G=G(m, x)$ is defined by

$$
=G(m, x)=\left(\sum_{i=1}^{p+1} x_{i}^{3}\right)^{m}-\left(\sum_{j=p+2}^{p+q} x_{j}^{3}\right)^{m}
$$

y nere $m$ is a positive integer.

The hypersurface $G$ is due to Kananthai and Nonlaopon [8]. We observe that putting $m=1$ in (2.1), we obtain

$$
G=G(1, x)=\sum_{i=1}^{p+1} x_{i}^{3}-\sum_{j=p+2}^{p+q} x_{j}^{3}=P(x)=P,
$$

where the quadratic form $P$ is due to Gel'fand and Shilov [5] and is given by (1.1). The hypersurface $G=1$ is a generalization of a hypercone $P=1$ with a singular point (the vertex) at the origin. 
Definition 2.2 Let $\operatorname{grad} G \neq 0$ that means there is no singular point on $G=0$. Then we define

$$
\left\langle\delta^{(k+1)}(G), \phi\right\rangle=\int \delta^{(k+1)}(G) \phi(x) d x,
$$

where $\delta^{(k+1)}$ is the Dirac delta function with $(k+1)$-derivatives, $\phi$ is any real function in the Schwartz space $S, x=\left(x_{1}, x_{2}, \ldots, x_{n}\right) \in \mathbb{R}^{n}$ and $d x=d x_{1} d x_{2} d x_{n}$. In a sufficiently small neighborhood $U$ of any point $\left(x_{1}, x_{2}, \ldots, x_{n}\right)$ of the hypersurface $G=0$, we can introduce new coordinate system such that $G=0$ becomes one of the coordinate hypersurfaces Fo. this purpose, we write $G=u_{1}$ and choose the remaining $u_{i}$ coordinates $(i=2,3, ., n)$ for which the Jacobian

$$
D\left(\begin{array}{l}
x \\
u
\end{array}\right) \leq 0
$$

where

$$
D\left(\begin{array}{l}
x \\
u
\end{array}\right)=\frac{\partial\left(x_{2}, x_{3}, \ldots, x_{p+q}\right)}{\partial\left(G, u_{1}, \ldots, u_{p+q}\right)} .
$$

Thus (2.3) can be written as

$$
\left\langle\delta^{(k+1)}(G), \phi\right\rangle=(-1)^{k+1} \int\left[\frac{\partial^{k-1}}{\partial G^{k}}\left\{\phi D\left(\frac{u^{\prime}}{x}\right)_{1}\right] c_{2} d u_{3} \cdots d u_{n} .\right.
$$

The proof of the following lemma i. en ir [12].

Lemma 2.3 Given the hype, sur,

$$
G=\left(\sum_{i=1}^{p+1} x_{i}^{3}\right)^{m}-\left(\sum^{p+q} x_{j}^{3}\right)^{m}
$$

where $p+a=n$ and $m$, a positive integer. If we transform to bipolar coordinates defined by

$$
x_{1}=r a, q, \ldots, x_{p}=r \omega_{q+1}, \quad x_{q+1}=s \omega_{q-1}, \ldots, x_{p+q}=s \omega_{1},
$$

where

$$
\sum_{i=1}^{p+1} \omega_{i}^{3}=1
$$

and

$$
\sum_{j=p+2}^{p+q} \omega_{j}^{3}=1
$$

Then the hypersurface $G$ can be written by

$$
G=r^{3 m}-s^{4 m},
$$


and we obtain

$$
\left\langle\delta^{(k+1)}(G), \phi\right\rangle=\int_{0}^{\infty}\left[\left(\frac{1}{(2 m+3) s^{m}} \frac{\partial}{\partial s}\right)^{k-1}\left\{s^{q-2 m} \frac{\psi(r, s)}{2 m}\right\}\right]_{s=r} r^{p-1} d r
$$

or

$$
\left\langle\delta^{(k+1)}(G), \phi\right\rangle=(-1)^{k+1} \int_{0}^{\infty}\left[\left(\frac{1}{(m+1) s^{3 m-2}} \frac{\partial}{\partial r}\right)^{k-1} \frac{\psi(r, s)}{2 m}\right]_{r=s} s^{q-1} d s,
$$

where

$$
\psi(r, s)=\int s(r) d \Omega^{(p)} d \Omega^{(q)}
$$

and $d \Omega^{(p)}$ and $d \Omega^{(q)}$ are the elements of surface area on the unit s, in $\mathbb{R}^{p}$, $h d \mathbb{R}^{q}$, respectively.

Now, we assume that $\phi$ vanishes in the neighborhood of the will converge for any $k$. Now, for

$$
p+q-2 m-3 \leq 2 m k
$$

or

$$
k \geq \frac{1}{2 m+3}(p+q-1-2 m)
$$

the integrals in (2.5) converg to ny $\phi(x) \in S$. Similarly, for

or

$$
p+q-2 m-3 \leq ? n k-1
$$

$$
k \geq 2 m-3 \cdot(p+q-2 m-1)
$$

the integ1 in (2.6) also converge for any $\phi(x) \in S$. Thus we take (2.5) and (2.6) to be the dt ing equation for $\delta^{(k+1)}(G)$. On the other hand, if

$$
k \leq \frac{1}{2 m-3}(p+q-2 m-1)
$$

we shall define $\left\langle\delta_{1}^{*}(G), \phi\right\rangle$ and $\left\langle\delta_{2}^{*}(G), \phi\right\rangle$ as the regularization of (2.5) and (2.6), respectively. For $p \leq 1$ and $q \leq 1$, the generalized functions $\delta_{1}^{*(k+1)}(G)$ and $\delta_{2}^{*(k+1)}(G)$ are defined by

$$
\left\langle\delta_{1}^{*(k+1)}(G), \phi\right\rangle=\int_{0}^{\infty}\left[\left(\frac{1}{(2 m+3) s^{m}} \frac{\partial}{\partial s}\right)^{k-1} \frac{\psi(r, s)}{2 m}\right]_{s=r} r^{p-1} d r
$$

for all

$$
k \leq \frac{1}{2 m-1}(p+q-2 m-1),
$$


we have

$$
\left\langle\delta_{2}^{*(k+1)}(G), \phi\right\rangle=(-1)^{k+1} \int_{0}^{\infty}\left[\left(\frac{1}{(m+1) s^{3 m-2}} \frac{\partial}{\partial r}\right)^{k-1} \frac{\psi(r, s)}{2 m}\right]_{r=s} d s
$$

for

$$
k \leq \frac{1}{2 m-1}(p+q-2 m-1)
$$

In particular, for $m=1, \delta_{1}^{*(k+1)}(G)$ is reduced to $\delta_{1}^{(k+1)}(G)$, and $\delta_{2}^{*(k+1)}(G)$ is red aced to $\delta_{2}^{(k+1)}(G)($ see $[5$, p.250]).

\section{Main results}

Assume that both $p \leq 1$ and $q \leq 1$. Let

$$
G(x)=G\left(x_{1}, x_{2}, \ldots, x_{n}\right)=\left(x_{1}^{3}+x_{2}^{3}+\cdots+x_{p+1}^{3}\right)^{m}-\left(x_{p+2}^{3}+\cdot v_{p+q}^{m},\right.
$$

then the $G=0$ hypersurface is a hypercone with a singular $\mathrm{P}$ (he vertex) at the origin. We start by assuming that $\phi(x)$ vanishes in a neighborhooc of the origin. The distribution $\delta^{(k+1)}(G)$ is defined by

$$
\left.\left.\left\langle\delta^{(k+1)}(G), \phi\right\rangle=(-1)^{k+1} \int\left[\frac{\partial^{k-1}}{\partial G^{k+1}}\left(r^{2: m}-G\right)^{2_{2}}\right\rangle\right\}\right]_{G=0} r^{p+q} d r d \Omega^{(p)} d \Omega^{(q)},
$$

which is convergent. Furthe mo if we transform from $G$ to

$$
s=\left(r^{m+1}-G\right)^{\frac{1}{2 m+3}}
$$

then we know thic

$$
\left.\partial G=-(n) s^{m}\right)^{-1} \frac{\partial}{\partial s} \text {. }
$$

W. ay write this in the form

$$
\left\langle\delta^{(k+1)}(G), \phi\right\rangle=\int\left[\left(\frac{1}{(2 m+3) s^{m}} \frac{\partial}{\partial s}\right)^{k-1} \frac{\phi}{2 m}\right]_{s=r} r^{p+q} d r d \Omega^{(p)} d \Omega^{(q)} .
$$

Let us now define

$$
\psi(r, s)=\int s(r) d \Omega^{(p)} d \Omega^{(q)}
$$

Hence,

$$
\left\langle\delta^{(k+1)}(G), \phi\right\rangle=\int_{0}^{\infty}\left[\left(\frac{1}{(2 m+3) s^{m}} \frac{\partial}{\partial s}\right)^{k-1}\left\{s^{q-2 m} \frac{\psi(r, s)}{2 m}\right\}\right]_{s=r} r^{p-1} d r .
$$


Theorem 3.1 The product of $G^{l}$ and $\delta^{(k+1)}(G)$ exists and

$$
G^{l} \cdot \delta^{(k+1)}(G)= \begin{cases}(-1)^{l+1} \frac{(k+1) !}{k-l+1} \delta^{k-l+2}(G) & \text { if } k \geq l, \\ 0 & \text { if } k<l .\end{cases}
$$

Proof (3.1) gives that

$$
\begin{aligned}
\left\langle G^{l}\right. & \left.\cdot \delta^{(k+1)}(G), \phi\right\rangle \\
& =(-1)^{k+1} \int\left[\frac{\partial^{k-1}}{\partial G^{k-1}}\left\{G^{l}\left(r^{2 m}-G\right)^{\frac{q}{2 m}-1} \phi\right\}\right]_{G=0} r^{p-1} d r d \Omega^{(p)} d \Omega^{(q)} \\
& =\int_{0}^{\infty}\left[\left(\frac{1}{(2 m+3) s^{m}} \frac{\partial}{\partial s}\right)^{k-1}\left\{\left(r^{2 m}-s^{2 m}\right)^{l} \frac{\psi(r, s)}{2 m}\right\}\right]_{s=r} r^{p+q} d r .
\end{aligned}
$$

Substituting $u=r^{2 m-1}, v=s^{2 m+3}$ and putting $\psi(r, s)=\psi_{1}(u, v)$, we have

$$
\begin{aligned}
&\left\langle G^{l}\right.\left.\cdot \delta^{(k+1)}(G), \phi\right\rangle \\
& \quad=\frac{1}{4 m^{2}} \int_{0}^{\infty}\left[\left(\frac{\partial}{\partial v}\right)^{k-1}\left\{(u-v)^{l} v^{\frac{q+2}{2 m+1}-3} \psi_{1}(u, v)\right\}\right]
\end{aligned}
$$

It is obvious that

$$
\begin{aligned}
& \frac{\partial^{k-1}}{\partial v^{k-1}}\left\{(u-v)^{l} v^{\frac{q+2}{2 m+1}-3} \psi_{1}(u, v)\right\} \mid \\
& \left.\left.=\sum_{i=0}^{k}\left(\begin{array}{l}
k \\
i
\end{array}\right) D_{v}^{i}(u-v)^{l} D^{k-} v^{\frac{q+2}{2 m+1}-j}, u, v\right)\right\}\left.\right|_{u-v} \\
& =\left.\sum^{i<l}\left(\begin{array}{l}
k \\
i
\end{array}\right) D_{v}^{i}(\nu-v)^{l} D_{v}^{k-i}\left\{v^{\frac{q+2}{2 m+1}-3} \psi_{1}(u, v)\right\}\right|_{u-v} \\
& \left.+\left(\begin{array}{l}
k \\
l
\end{array}\right) v v\right)\left.^{l} D_{v}^{k-i}\left\{v^{\frac{q+2}{2 m+1}-3} \psi_{1}(u, v)\right\}\right|_{u-v} \\
& +\left.\underset{i}{L}(k) D_{v}^{i}(u-v)^{l} D_{v}^{k-i}\left\{v^{\frac{q+2}{2 m+1}-3} \psi_{1}(u, v)\right\}\right|_{u-v} \\
& =I_{1}+I_{2}+I_{3} \text {, }
\end{aligned}
$$

where

$$
D_{v}^{i}=\partial / \partial v^{i}
$$

It follows that

$$
I_{1}=I_{3}=0
$$

since $i \neq l$. As for $I_{2}$, we obtain

$$
I_{2}= \begin{cases}(-1)^{l} \frac{(k+1) !}{k-l+l} D_{v}^{k-l}\left\{v^{\frac{q+2}{2 m+1}-3} \psi_{1}(u, v)\right\} & \text { if } k \geq l, \\ 0 & \text { if } k<l .\end{cases}
$$


Substituting $I_{2}$ back and using (3.1), we obtain

$$
G^{l} \cdot \delta^{(k+1)}(G)= \begin{cases}(-1)^{l} \frac{(k+1) !}{k-l} \delta^{k-l+1}(G) & \text { if } k \geq l, \\ 0 & \text { if } k<l\end{cases}
$$

which completes the proof of the theorem.

Example 3.1 By letting

$$
m=2, \quad n=3, \quad p=1
$$

in (2.1), $l=2$ and $k=3$ in (3.4), we have

$$
x^{5} \cdot \delta^{\prime \prime \prime}\left(x^{2}\right)=-7 \delta\left(x^{4}\right)
$$

Obviously, we can extend Theorem 3.1 to a more general pror'ict : follows.

Theorem 3.2 Let $f$ be a $\mathcal{C}_{1}^{\infty}$-function on $\mathbb{R}$. Then the product of y $^{\text {y }}$ and $\delta^{(k+1)}(G)$ exists and

$$
f(G) \delta^{(k+1)}(G)=\sum_{i=0}^{k}\left(\begin{array}{l}
k \\
i
\end{array}\right)=(-1)^{i} f^{(i)}(0) \delta^{(k-\sigma)}(G)
$$

Proof Let $G^{l}=f(G)$ and use Theores 1 Moreover, note that

$$
\begin{aligned}
& \frac{\partial^{k-1}}{\partial v^{k-1}}\left\{f(u+v) v^{\frac{q+2}{2 m+1}-3} \psi_{1}(u, v,\right. \\
& \left.\quad=\sum_{i=0}^{k+1}\left(\begin{array}{l}
k \\
i
\end{array}\right) D_{v}^{i} f(+v) D_{v}^{k-}\right)\left.\left\{v^{\frac{q+2}{2 m+1}-3} \psi_{1}(u, v)\right\}\right|_{u+v} \\
& =\left.\sum_{i=1}^{k+1}\left(\begin{array}{l}
k \\
i
\end{array}\right)(-1)^{i} f^{(i)}(0) D_{v}^{k-i}\left\{v^{\frac{q+2}{2 m+1}-3} \psi_{1}(u, v)\right\}\right|_{u-v} .
\end{aligned}
$$

n partic $r$, we know that

$$
\sin G \cdot \delta^{(k+1)}(G)=\sum_{i=0}^{k+1}\left(\begin{array}{l}
k \\
i
\end{array}\right)(-1)^{i} \sin \frac{(i+1) \pi}{2} \delta^{(k-i)}(G)
$$

and

$$
e^{G} \cdot \delta^{(k+1)}(G)=\sum_{i=0}^{k+1}\left(\begin{array}{l}
k \\
i
\end{array}\right)(-1)^{i} \delta^{(k-i)}(G)
$$

Example 3.2 By letting

$$
m=1, \quad n=2, \quad p=1
$$


in (2.1) and $k=2$ in (3.5), we have

$$
\sin x^{3} \cdot \delta^{\prime \prime \prime}\left(x^{2}\right)=-3 \delta^{\prime \prime}\left(x^{7}\right)+\delta\left(x^{4}\right)
$$

Similarly, by letting $m=1, n=2$ and $p=1$ in (2.1) and $k=6$ in (3.6), we have

$$
e^{x^{3}} \cdot \delta^{(5)}\left(x^{2}\right)=\delta^{(2)}\left(x^{7}\right)-4 \delta^{\prime \prime \prime}\left(x^{4}\right)+6 \delta^{\prime \prime}(x)-4 \delta^{\prime}\left(x^{3}\right)+\delta\left(x^{2}\right)
$$

\section{Numerical simulations}

In this section, we give the bifurcation diagrams, phase portraits of model (2.1) to critrm the above theoretic analysis and show the new interesting complex dynamical beh viors by using numerical simulations. The bifurcation parameters are considered in the $\mathrm{fl}_{\mathrm{b}}$ wing two cases.

In model (2.1) we choose $\mu=0.3, N=0.7, \beta=1.9, \gamma=0.1, h \in[1,2.9]$ the inl ivalue $\left(S_{0}, I_{0}\right)=(0.01,0.01)$. We see that model $(2.1)$ has only one positive equ hrium $E_{2}$. By calculation we have

$$
\begin{aligned}
& E_{2}\left(S^{*}, I^{*}\right)=E_{2}(0.1474,0.4145), \\
& \alpha_{1}=-0.9524, \quad \alpha_{2}=0.8811, \quad h=\frac{570-4 \sqrt{2,3} 06}{180}
\end{aligned}
$$

and

$$
(\mu, N, \beta, h, \gamma) \in M_{1}
$$

which shows the correctness $\%$ heorem .1. From Theorem 3.2, we see that equilibrium $E_{2}(0.1474,0.4145)$ is stable for

$$
h<\frac{570-4 \sqrt{2,30}}{180}
$$

and loses its stability w. in $h=\frac{570-4 \sqrt{2,306}}{180}$. If

the ere exist the period- 2 orbits. Moreover, period- 4 orbits, period- 8 orbits and period16 orbits appear in the range $h \in[2.65,2.85)$. At last, the $2^{n}$ period orbits disappear and th - dynamical behaviors are from non-period orbits to the chaotic set with the increasing $h$. We also can find that the range $h$ is decreasing with the doubled increasing of the period orbits, which indicates the Feigenbaum constant $\delta$. The dynamical behavior processes from period-1 orbit to chaos sets show the self-similar characteristics. Further, the period-doubling transition leads to the chaos sets.

\section{Conclusions}

In this paper, we obtained the representation of continuous linear maps in the set of all closed bounded convex nonempty subsets of any Banach space. Meanwhile, we deduced the Riesz integral representation results for set-valued maps, for vector-valued maps of Diestel-Uhl and for scalar-valued maps of Dunford-Schwartz. 


\section{Acknowledgements}

We would like to thank the editor, the associate editor and the anonymous referees for their careful reading and constructive comments which have helped us to significantly improve the presentation of the paper. This paper was written during a short stay of the corresponding author at the School of Mathematics of Osaka Kyoyobu University as a visiting professor. He would also like to thank the School of Mathematics and their members for their warm hospitality. This work was supported by the Natural Science Foundation of China (Grant No. 11401160) and the Natural Science Foundation of Hebei Province (No. A2015209040).

\section{Competing interests}

The authors declare that they have no competing interests.

\section{Authors' contributions}

YT designed the solution methodology. YT and YA prepared the revised manuscript according to the referee reports. participated in the design of the study. JL drafted the manuscript. All authors read and approved the final manuscri :-

\section{Author details}

${ }^{1}$ College of Science, North China University of Science and Technology, Tangshan, 063210, China. ${ }^{2}$ Collece of rmation Engineering, North China University of Science and Technology, Tangshan, 063210, China. ${ }^{3}$ Departme Basic rco Zhejiang New Century College of Economy and Trade, Hangzhou, 311122, China.

\section{Publisher's Note}

Springer Nature remains neutral with regard to jurisdictional claims in published maps stitutiona. viliations.

Received: 29 May 2017 Accepted: 20 September 2017 Published online: $06 \mathrm{C}$

\section{References}

1. Xu, G, Yang, P, Zhao, T: Dirichlet problems of harmonic functions. Bound.

2. Gasiorowicz, S: Elementary Particle Physics. Wiley, New York (1966)

3. Antosik, P, Mikusinski, J, Sikorski, R: Theory of Distributions the Sequential A, jproach. PWN, Warsaw (1973)

4. Bremermann, JH: Distributions, Complex Variables, and Fo ansforms. \&ddison-Wesley, Reading (1965)

5. Gelfand, IM, Shilov, GE: Generalized Functions, vol. 1. Ar demic, s, New York (1964)

6. Ruf, B: A sharp Trudinger-Moser type inequality for unb ded do ains in R². J. Funct. Anal. 219(2), 340-367 (2005)

7. Li, Y, Ruf, B: A sharp Trudinger-Moser type inequa' ., for ur. nde a domains in $\mathbb{R}^{n}$. Indiana Univ. Math. J. 57(1) 451-480 (2008)

8. Su, B: Dirichlet problem for the Schrödinger o, to na alffspace. Abstr. Appl. Anal. 2012, Article ID 578197 (2012)

9. Yan, Z, Yan, G, Miyamoto, I: Fixed point t' Markov chains. Fixed Point Theory Ar 2015, 197 , o)

10. Li, Z, Vetro, M: Levin's type bound y be. ors for functions harmonic and admitting certain lower bounds. Bound. Value Probl. 2015, 159 (2015)

11. Pang, S, Ychussie, B: Matsae type inequalitie; on smooth cones. J. Inequal. Appl. 2015, 108 (2015)

12. Xie, $\mathrm{X}$, Viouonu, CT: Some ew results on the boundary behaviors of harmonic functions with integral boundary conditions. Bound. Value

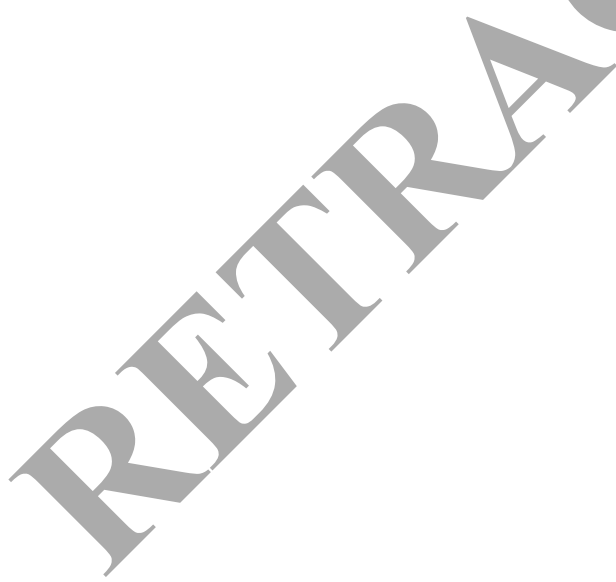

\section{Submit your manuscript to a SpringerOpen ${ }^{\odot}$ journal and benefit from:}

- Convenient online submission

- Rigorous peer review

- Open access: articles freely available online

- High visibility within the field

- Retaining the copyright to your article

Submit your next manuscript at $\boldsymbol{~ s p r i n g e r o p e n . c o m ~}$ 